\title{
Wormlike core-shell nanoparticles formed by co-assembly of double hydrophilic block polyelectrolyte with oppositely charged fluorosurfactant $\uparrow$
}

\author{
Miroslav Štěpánek, ${ }^{* a}$ Juraj Škvarla, ${ }^{a}$ Mariusz Uchman, ${ }^{a}$ Karel Procházka, ${ }^{a}$ Borislav Angelov, ${ }^{b}$ Lubomír

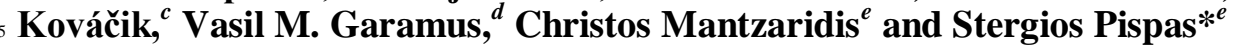

\author{
Received (in $X X X, X X X)$ Xth $X X X X X X X X X 20 X X$, Accepted Xth $X X X X X X X X X 20 X X$ \\ DOI: $10.1039 / \mathrm{b} 000000 \mathrm{x}$
}

Formation of polyelectrolyte-surfactant complexes (PE-S) between anionic polyelectrolyte, poly(sodium

2-sulfamate-3-carboxylate isoprene)-block-poly(ethylene oxide) (PSCI-PEO) and cationic

10 fluorosurfactant, $N$-(3,3,4,4,5,5,6,6,7,7,8,8,9,9,10,10,10-heptadecafluorodecyl)pyridinium chloride

(HFDPCl) was studied in alkaline aqueous solutions by static, dynamic and electrophoretic light scattering. The structure of the formed PE-S nanoparticles was investigated by SAXS, cryogenic transmission electron microscopy and atomic force microscopy. The results show that tendency of the fluorosurfactant to form elongated threadlike micelles drives the PE-S co-assembly to a flexible core-shell 15 cylindrical morphology with the core of the PE-S and the shell of the PEO blocks. Unlike other PE-S systems involving double hydrophilic polyelectrolytes, well-defined core-shell particles exist only in the narrow range of HFDPCl-to-PSCI units stoichiometric ratios corresponding to zero $\zeta$ potential of the aggregates.

\section{Introduction}

20 Complexes of polyelectrolytes with oppositely charged surfactants (PE-S) have attracted attention of many researchers in the past two decades due to general interest in nanostructured self-assembled systems, as well as due to pharmaceutical and other applications of such materials. ${ }^{1-3}$ PE-S are stabilized by

25 electrostatic interactions between the surfactant head groups and the polyelectrolyte side groups and by hydrophobic interactions between the hydrophobic backbone of the polyelectrolyte and the alkyl chains of the surfactant. It has been found that the oppositely charged surfactant condenses on the polyelectrolyte

30 chains and forms micelles at concentrations far below its critical micelle concentration $(\mathrm{cmc})$ in the absence of the polyelectrolyte $^{\mathbf{4 , 5}}$ and that water-insoluble stoichiometric PE-S with zero net charge form various water-insoluble ordered crystalline-like phases. ${ }^{6}$

35 Block copolymers consisting of a polyelectrolyte block and a neutral hydrophlic block (further referred to as double hydrophilic block polyelectrolytes, DHBE), such as poly(methacrylate)-block-poly(ethylene $\quad$ oxide $)^{7-9}$ or poly(acrylate)-block-poly(acrylamide) $)^{\mathbf{1 0 - 1 2}}$ co-assemble with 40 oppositely charged ionic surfactants into core-shell particles with the shell formed by the neutral hydrophilic block and the core formed by the insoluble PE-S of the polyelectrolyte block and the surfactant. SANS and SAXS measurements indicate that the core contains disordered densely packed surfactant micelles. ${ }^{10,11}$

45 Surfactants with perfluorocarbon tails have been a subject of study due to their favorable properties such as lower solubility in water, lower cmc and stronger surface activity as compared with their hydrocarbon analogs. ${ }^{\mathbf{1 3}}$ Surfactants containing perfluorinated alkyl chains differ in many respects from 50 hydrocarbon surfactants not only because the fluorocarbon tail is both hydrophobic and lipophobic but also because of its larger excluded volume as compared with hydrocarbons which causes fluorosurfactants to prefer self-assembly with a less curved interface, such as elongated micelles or vesicles. ${ }^{13}$ Although a 55 number of papers deal with complexes of homopolymers with fluorosurfactants, ${ }^{\text {14-18 }}$ and formation of co-assembled nanoparticles of block copolymers with fluorosurfactants in organic solvents have also been reported, ${ }^{\mathbf{1 9 , 2 0}}$ there is only a few studies on PE-S of DHBE with an oppositely charged ${ }_{60}$ fluorosurfactant in aqueous solutions. ${ }^{21}$

Here we report on the formation and structure of PE-S nanoparticles composed of an anionic DHBE, poly(sodium 2sulfamate-3-carboxylate isoprene)-block-poly(ethylene oxide), PSCI-PEO ${ }^{\mathbf{2 2}}$ and a cationic fluorosurfactant, N${ }_{65}(3,3,4,4,5,5,6,6,7,7,8,8,9,9,10,10,10 \quad$-heptadecafluorodecyl $)$ pyridinium chloride, $\mathrm{HFDPCl}$, in alkaline aqueous solutions. It has been reported that above certain critical concentration (which is dependent on ionic strength), HFDPCl undergoes the transition from spherical to elongated, threadlike micelles. ${ }^{23}$ In this paper, 70 we investigate how tendency of the surfactant to form elongated micelles influences the coassembly with DHBE. As a DHBE component of the studied PE-S complexes, we use PSCI-PEO, a biocompatible copolymer whose polyelectrolyte block contains both strong $\left(\mathrm{SO}_{3} \mathrm{H}\right)$ and weak $(\mathrm{COOH})$ acidic groups (similarly to 
heparin $^{\mathbf{2 4 , 2 5}}$ ) and its backbone is strongly hydrophobic due to the presence of unmodified poly(isoprene) units. The ability of PSCIPEO to form nanoparticles with various positively charged species has been widely studied. It has been reported that PSCI5 PEO copolymers form complexes with cationic polyelectrolytes and surfactants, ${ }^{\mathbf{2 6}}$ cationic vesicles, ${ }^{27}$ proteins with a high isoelectric point ${ }^{28}$ or bivalent metal cations. ${ }^{29}$

In this paper, the formation of PE-S between PSCI-PEO and $\mathrm{HFDPCl}$ is followed by a combination of static, dynamic and 10 electrophoretic light scattering. The structure of the formed nanoparticles is studied in detail using small angle X-ray scattering (SAXS), cryogenic transmission electron microscopy (Cryo-TEM) and atomic force microscopy (AFM).

\section{${ }_{15} 2$ Experimental}

\subsection{Materials}

The PSCI-PEO copolymer was prepared by the postpolymerization reaction of polyisoprene-block-poly(ethylene 20 oxide) (PI-PEO), prepared by living anionic polymerization, ${ }^{21}$ with chlorosulfonyl isocyanate. Details on the synthesis can be found in ref. 21. Weight average molar mass of the PI-PEO precursor by SEC was, $M_{\mathrm{w}}=17.4 \mathrm{~kg} \mathrm{~mol}^{-1}$, the PSCI-PEO sample composition by ${ }^{13} \mathrm{C}$ solid state NMR was 42,34 and 24 $25 \mathrm{~mol}$. \% for PEO units, PSCI units and unmodified polyisoprene units, respectively.

$\mathrm{HFDPCl}$ was synthesized from $1 \mathrm{H}, 1 \mathrm{H}, 2 \mathrm{H}, 2 \mathrm{H}$-perfluorodecyl iodide and pyridine following the procedures outlined in ref 30 .

PE-S complexes were prepared by mixing stock solutions of 30 PSCI-PEO and HFDPCl. Stock solutions of PSCI-PEO in $50 \mathrm{mM}$ aqueous sodium tetraborate $(\mathrm{pH} 9.3)$ and $\mathrm{HFDPCl}$ in deionized water were mixed under stirring and left to stand at least 24 hours for equilibration prior to measurements. PSCI-PEO concentration in the solutions was $1 \mathrm{mg} \mathrm{mL}^{-1}$.

35

\subsection{Methods}

Static and dynamic light scattering measurements were carried out with an ALV photometer (ALV, Langen, Germany) consisting of a $22 \mathrm{~mW}$ He-Ne laser, an ALV CGS/8F goniometer, 40 an ALV High QE APD detector and an ALV 5000/EPP multibit, multitau autocorrelator at $20^{\circ} \mathrm{C}$. The copolymer concentration in all solutions was, $c=1 \mathrm{mg} / \mathrm{mL}$.

Static light scattering (SLS) measurements of the corrected excess scattering intensity $I(q)$, of the copolymer solutions, as a 45 function of the magnitude of the scattering vector $q=\left(4 \pi n_{0} / \lambda\right) \sin (\theta / 2)\left(\theta\right.$ being the scattering angle, $n_{0}=1.332$ the refractive index of the solvent and $\lambda=632.8 \mathrm{~nm}$ the wavelength of the incident light) were treated by fitting the data in the angular range from $30^{\circ}$ to $90^{\circ}$ to the Guinier equation,

$50 \quad \ln \frac{I(q)}{I(0)}=-\frac{1}{3} R_{\mathrm{g}}^{2} q^{2}$,

to obtain the forward scattering intensity, $I(0)$, and the radius of gyration, $R_{\mathrm{g}}$.

Dynamic light scattering measurements were evaluated by fitting the measured normalized time autocorrelation function of
55 the scattered light intensity, $g^{(2)}(t)$, related to the electric field autocorrelation function, $g^{(1)}(t)$, by the Siegert relation, $g^{(2)}(t)=1+\beta\left|g^{(1)}(t)\right|^{2}$, where $\beta$ is the coherence factor. A constrained regularization algorithm (CONTIN) provided the distribution of relaxation times $\tau, A(\tau)$, as the inverse Laplace transform of $g^{(1)}(t)$ 60 function

$$
g^{(1)}(t)=\int_{0}^{\infty} A(\tau) \exp \left(-\frac{t}{\tau}\right) \mathrm{d} \tau,
$$

The $A(\tau)$ distributions were recalculated to the distributions of apparent hydrodynamic radii, $R_{\mathrm{H}}^{\text {app }}$, assuming the apparent diffusion coefficient $D^{\text {app }}=1 / \tau q^{2}$ and using the Stokes-Einstein ${ }_{65}$ formula, $R_{\mathrm{H}}{ }^{\text {app }}=k_{\mathrm{B}} T / 6 \pi \eta D^{\text {app }}$, where $k_{\mathrm{B}}$ is the Boltzmann constant, $T$ the temperature and $\eta$ the solvent viscosity.

Electrophoretic light scattering measurements were carried out with a Nano-ZS Zetasizer (Malvern Instruments, U.K.) לPotential values were calculated from electrophoretic mobilities 70 (averages of 15 up to 100 measurements) using the Smoluchowski approximation.

SAXS experiments were performed on the P12 BioSAXS beamline at the storage ring PETRA III of the Deutsche Elektronen Synchrotron (DESY, Hamburg, Germany) at $20{ }^{\circ} \mathrm{C}$ 75 using a Pilatus $2 \mathrm{M}$ detector and synchrotron radiation with the wavelength of $\lambda=0.1 \mathrm{~nm}$. The sample-detector distance was $3 \mathrm{~m}$, allowing for measurements in the $q$-range interval from 0.11 to $4.4 \mathrm{~nm}^{-1}$. The $q$ range was calibrated using the diffraction patterns of silver behenate. The experimental data were normalized to the 80 incident beam intensity, corrected for non-homogeneous detector response, and the background scattering of the solvent was subtracted. The solvent scattering was measured before and after the sample scattering to control for possible sample holder contamination. Eight consecutive frames comprising the 85 measurement of the solvent, sample, and solvent were performed. No measurable radiation damage was detected by a comparison of eight successive time frames with $15 \mathrm{~s}$ exposures. The final scattering curve was obtained using the program PRIMUS by averaging the scattering data collected from the different frames. ${ }_{90}$ The automatic sample changer for sample volume $15 \mu \mathrm{L}$ and filling cycle of $20 \mathrm{~s}$ was used.

Cryo-TEM measurements were carried out using a Tecnai G2 Sphera 20 electron microscope (FEI Company, Hillsboro, OR, USA) equipped with a Gatan 626 cryo-specimen holder (Gatan, ${ }_{95}$ Pleasanton, CA, USA). The samples for cryo-TEM were prepared by plunge-freezing as described earlier. ${ }^{31}$ Briefly, $3 \mu \mathrm{L}$ of the sample solution were applied to an electron microscopy grid covered with perforated carbon supporting film (C-flat $2 / 2-2 \mathrm{C}$, Electron Microscopy Sciences) glow discharged for $20 \mathrm{~s}$ with 5 $100 \mathrm{~mA}$ current. Most of the sample was removed by blotting (Whatman no. 1 filter paper) for approximately $1 \mathrm{~s}$, and the grid was immediately plunged into liquid ethane held at $-183^{\circ} \mathrm{C}$. The grid was then transferred without rewarming into the microscope. Images were recorded at the accelerating voltage of $120 \mathrm{kV}$ and 105 microscope magnification ranging from $5000 \times$ to $14500 \times$ using a Gatan UltraScan 1000 slow scan CCD camera (giving a final pixel size from 2 to $0.7 \mathrm{~nm}$ ) and low dose mode with the electron dose not exceeding 1500 electrons per $\mathrm{nm}^{2}$. Typical value of applied underfocus ranged between 0.5 to $2 \mu \mathrm{m}$. The applied 110 blotting conditions resulted in the specimen thickness varying between 100 to ca. $300 \mathrm{~nm}$. 
AFM measurements were performed in the tapping mode under ambient conditions using a commercial scanning probe microscope, Digital Instruments NanoScope dimensions 3, equipped with a Nanosensors silicon cantilever with a typical 5 spring constant of $40 \mathrm{~N} \mathrm{~m}^{-1}$. Polymeric micelles were deposited on a fresh (i.e., freshly peeled out) mica surface (flogopite, theoretical formula $\mathrm{KMg}_{3} \mathrm{AlSi}_{3} \mathrm{O}_{10}(\mathrm{OH})_{2}$, the Geological Collection of the Charles University in Prague, Czech Republic) by a fast dip coating in a PSCI-PEO/HFDPCl aqueous solution ( $c$ 10 ca. $\left.10^{-2} \mathrm{~g} \mathrm{~L}^{-1}\right)$. After the evaporation of water, the sample was dried in vacuum oven at ambient temperature for ca. 5 hours.

\section{Results and discussion}

\section{3.1 Light scattering study of PE-S aggregates formation}

At first, the formation of PE-S in PSCI-PEO/HFDPCl system has been studied by static, dynamic and electrophoretic light scattering. Fig. 1 shows the dependences of the forward scattering intensity, $I(0)$ (curve 1 ), and the gyration radius, $R_{\mathrm{g}}$ (curve 2 ) of 20 the PE-S aggregates formed in PSCI-PEO/HFDPCl alkaline aqueous solutions as functions of stoichiometric ratio between the surfactant and the polyelectrolyte units of PSCI, $\beta=$ $[\mathrm{HFDPCl}] /[\mathrm{SCI}]$. The results indicate that large aggregates are present already in pure PSCI-PEO alkaline solution $\left(R_{\mathrm{g}}=118\right.$ $25 \mathrm{~nm}$ ) due to hydrophobic interactions between unmodified isoprene units. For low $\beta$ up to 0.36 , both average size of the scatterers in the PSCI-PEO/HFDPCl solution and forward scattering intensity increase with the increasing stoichiometric ratio as the formation the PE-S complex further promotes 30 aggregation of PSCI-PEO chains. When the amount of the surfactant is further increased, $R_{\mathrm{g}}$ decreases (while $I(0)$ still grows) because the polyelectrolyte chains collapse due to charge neutralization by the bound surfactant micelles and consequent decrease of the electrostatic repulsion between the PSCI 35 segments.

The above mentioned charge neutralization can be followed by electrophoretic light scattering measurements. Inset in Fig. 1 shows the $\zeta$ potential of the PSCI-PEO/HFDPCl system, which reflects the effective net charge of the formed PE-S aggregates, as 40 a function of $\beta$. The $\zeta$ values increase from negative to positive as the increasing amount of cationic surfactant is bound to the complex with the polyelectrolyte. The zero $\zeta$ potential (and thus the zero effective charge of the PE-S nanoparticles) appears at $\beta$ $=0.71$, very close to the composition at which the gyration radius

45 of the particles reaches its minimum value. On the other hand, the zero effective charge indicated by the $\zeta$ potential measurement is reached at a much lower amount of the added surfactant than that expected from the stoichiometry (as the charge of the SCI unit in alkaline solution should be -2 , the PE-S complex should have the

50 zero net charge at $\beta=2$ ). However, since the $\zeta$ potential value reflects rather the surface charge of the nanoparticles than their overall charge, this difference can be explained by the assumption that the charge ratio is shifted in favour of positive charges close to the surface of the aggregates as compared with their inner 55 parts, most probably due to adsorption of excess surfactant cations. Another factor which can influence the charge balance in the system is possible suppression of the ionization of carboxylic protons in the neighbourhood of $\mathrm{SO}_{3}{ }^{-}$groups so that the effective charge per one SCI unit is lower than -2 .

60 The presence of stable nanoparticles with the zero $\zeta$ potential in the PSCI-PEO/HFDPCl system indicates that the particles are not stabilized electrostatically and that a steric stabilization takes place, suggesting that PEO blocks segregate from the insoluble PE-S complex and the nanoparticles have a core-shell structure. 65

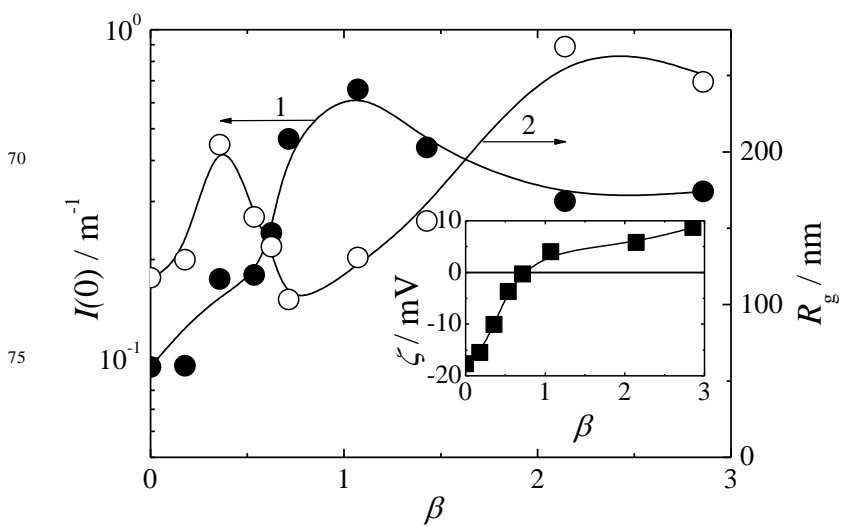

80 Fig. 1 Forward scattering intensity, $I(0)$ (curve 1), and gyration radius, $R_{\mathrm{g}}$ (curve 2) of PSCI-PEO/HFDPCl aggregates as functions of the stoichiometric ratio, $\beta$. Inset: $\zeta$ potential of $\mathrm{PSCI}-\mathrm{PEO} / \mathrm{HFDPCl}$ aggregates as a function of $\beta$.

85 For $\beta>0.71$, the gyration radius increases again suggesting that the compact nanoparticles are disrupted and looser aggregates are formed again. This assumption is further supported (i) by the dependence of $I(0)$ which slightly decreases with increasing $\beta$ above $\beta=1.07$, and (ii) by the $\zeta$ potential 90 dependence which shows that the aggregates overcharge, so that increasing electrostatic repulsion may contribute to the disruption of the nanoparticles and formation of loose aggregates.

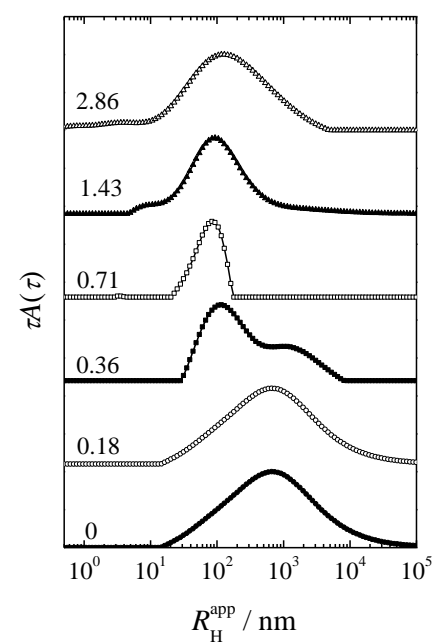

110 Fig. 2 DLS distributions of apparent hydrodynamic radii at the scattering angle, $\theta=90^{\circ}$, for PSCI-PEO/HFDPCl aggregates. The stoichiometric ratios, $\beta$, are given above the corresponding curves.

Fig. 2 shows distributions of apparent hydrodynamic radii of 
aggregates present in PSCI-PEO/HFDPCl solutions at different compositions of the PE-S complexes. In pure PSCI-PEO and at $\beta$ $=0.18$, the distributions are very broad, indicating formation of polydisperse aggregates. At $\beta=0.36$, the distribution becomes 5 clearly bimodal due to the coexistence of larger, loose aggregates with smaller, compact particles with a narrower size distribution which form as a result of charge neutralization. The scattering from the latter particles dominates at $\beta=0.71$, where also the minimum of $\mathrm{R}_{\mathrm{H}}{ }^{\text {app }}$ is reached at the same point as in the case of 10 the minimum $R_{\mathrm{g}}$ and the zero $\zeta$ potential. For higher $\beta$, the width of the $\mathrm{R}_{\mathrm{H}}{ }^{\text {app }}$ distribution is further increased indicating the disruption of the nanoparticles.

\section{3.3 SAXS study of PE-S aggregates structure}

In order to obtain information about the structure of aggregates and particles formed in the PSCI-PEO/HFDPCl mixed system, we have contacted small-angle X-ray scattering experiments. Fig. 3 depicts SAXS data for stoichiometries of the PE-S 20 corresponding to pure PSCI-PEO solution $(\beta=0)$, to loose aggregates prior to the formation of insoluble PE-S $(\beta=0.36)$, to compact PE-S nanoparticles $(\beta=0.71)$ and to loose aggregates formed by disruption of compact PE-S nanoparticles in excess of HFDPCl $(\beta=1.43)$.

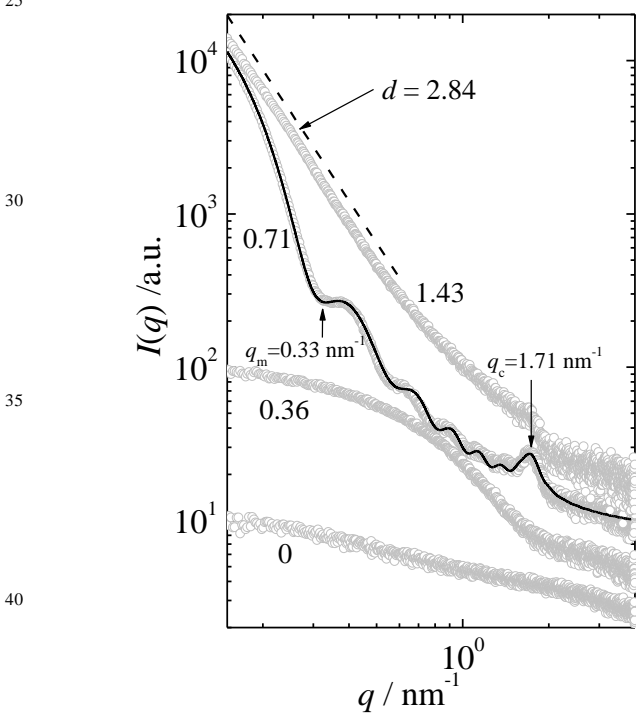

45 Fig. 3. SAXS of PSCI-PEO/HFDPCl aggregates. The stoichiometric ratios, $\beta$, are given at the corresponding curves. The solid line is the fit of Eqn. (3) to the data for $\beta=0.71$, the dashed line is a function proportional to $q^{-2.84}$

50 While scattering from PSCI-PEO coils in the pure copolymer solution is too weak for analysis of the curve, after addition of the surfactant, a dominant Guinier scattering contribution with the gyration radius $R_{\mathrm{g}}=2.04 \pm 0.10 \mathrm{~nm}$ appears for $\beta=0.36$. This behaviour can be attributed to the formation of spherical HFDPCl 55 micelles attached to PSCI chains.

A large increase in the scattering intensity in the low $q$ range after increasing $\beta$ from 0.36 to 0.71 corresponds to the formation of large compact PE-S nanoparticles. The scattering curve for the compact particles at $\beta=0.71$ (the minium size, the zero $\zeta$ 60 potential) exhibits oscillations characteristic for compact scatterers with spherical or cylindrical symmetry, suggesting formation of spherical or wormlike core/shell particles. The positions of local maxima and minima of the form factors for spheres and cylinders are associated with the radii of the objects, ${ }_{65} R$; for the first minimum $q_{\mathrm{m}}, q_{\mathrm{m}} R=4.46$ for the monodisperse spheres and $q_{\mathrm{m}} R=3.83$ for the long monodisperse cylinders. In the measured scattering curve, $q_{\mathrm{m}}=0.33 \mathrm{~nm}^{-1}$, which would correspond to the radius of $13.5 \mathrm{~nm}$ for the spherical geometry and $11.5 \mathrm{~nm}$ for the cylindical geometry. The assumption that the 70 PE-S complex forms a compact self-assembly is supported by the scattering behaviour in the high $q$-range which reflects close packing of the surfactant micelles in the formed PE-S complex, whose characteristic spacing $l$ manifests itself as a structure peak with the maximum at $q_{\mathrm{c}}=2 \pi / l$. (Presence of similar peaks was 75 reported for several other PE-S systems, both in the bulk ${ }^{6}$ and in the cores of DHBE core-shell nanoparticles. ${ }^{\mathbf{1 0}, 11}$ ) In section 3.5, we propose a model for the scattering behaviour of the PSCI$\mathrm{PEO} / \mathrm{HFDPCl}$ system at $\beta=0.71$, consistent with the results of Cryo-TEM measurements (section 3.4).

80 With further increasing amount of the surfactant, the oscillations disappear, indicating loss of the spherical or cylindrical shape. At $\beta=1.43$, the scattering curve exhibits a power law behavior, $I(q) \sim q^{-d}$, with the exponent $d=2.84$ in the $q$ range $0.15-0.4 \mathrm{~nm}^{-1}$ corresponding to mass fractal aggregates 85 of a sponge-like structure with the fractal dimension 2.84 on the lengthscale of ca. $10-40 \mathrm{~nm}$. It is worth mentioning that the SAXS curve for the fractal aggregate retains the structure peak at $q=1.71 \mathrm{~nm}^{-1}$, which means that the packing of surfactant micelles in the aggregate is preserved, even though the 90 broadening of the peak indicates that the correlation length within the packed complex of HFDPCl micelles and PSCI chains is decreased as compared with the compact particles at $\beta=0.71$.

\section{3.4 Cryo-TEM and AFM imaging of PE-S aggregates}

Since SAXS measurements indicated that at the stoichiometric ratio $\beta=0.71$, PSCI-PEO and $\mathrm{HFDPCl}$ co-assemble in spherical and/or cylindrical nanoparticles, the PSCI-PEO/HFDPCl system of this composition was imaged by Cryo-TEM and AFM. Cryo100 TEM (Fig. 4a) shows (i) wormlike nanoparticles with the crosssection radius of about $13 \mathrm{~nm}$ and length of several hundreds $\mathrm{nm}$ and (ii) spherical objects with a similar radius as the cross-section radius of the wormlike particles, which can either be spherical particles or short cylindrical particles imaged along their 105 longitudinal axes. The presence of elongated particles can be explained by the fact that $\mathrm{HFDPCl}$ undergoes a transition from spherical to threadlike micelles, ${ }^{\mathbf{2 3}}$ which, occuring simultaneously with the co-assembly of the surfactant cations with PSCI blocks, can lead to the formation of wormlike core-shell PE-S particles. 110 (A few isolated threadlike HFDPCl micelles can also be observed in the PSCI-PEO/HFDPCl system at $\beta=0.71$. The detail of a Cryo-TEM micrograph showing such a micelle is given as Supplementary Information.)

Fig. $4 \mathrm{~b}$ is an AFM scan of PE-S nanoparticles at $\beta=0.71$ in 115 dry state, deposited on mica surface from diluted aqueous 
solution $\left(c=10^{-2} \mathrm{mg} / \mathrm{mL}\right)$. Instead of isolated clusters, interconnected particles form a continuous two-dimensional network with both variable height and width on the substrate surface. It is noteworthy that the minimum width of the network 5 is about $30 \mathrm{~nm}$, which is in accordance with the dimensions of the worms. This observation suggests that at these points the junctions of the network are formed by single wormlike nanoparticles.

10
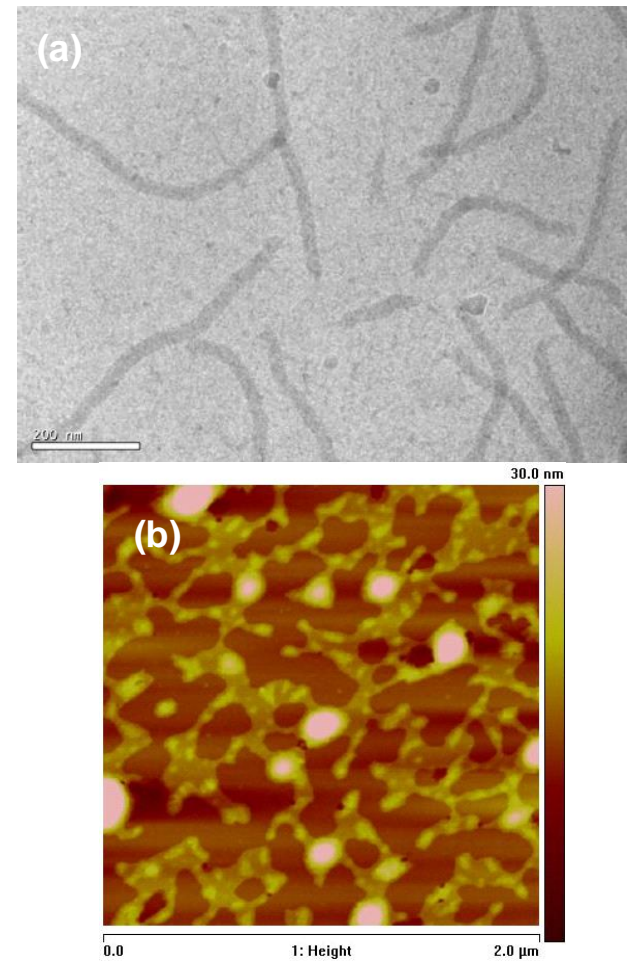

Fig. 4 Images of PSCI-PEO/HFDPCl nanoparticles at the stoichiometric ratio, $\beta=0.71$ : (a) Cryo-TEM (scale bar $=200 \mathrm{~nm})$, (b) AFM $\left(2 \times 2 \mu \mathrm{m}^{2}\right.$ 30 scan)

\subsection{SAXS model for compact nanoparticles}

In order to describe the SAXS scattering behaviour of the $\mathrm{PEO} / \mathrm{HFDPCl}$ system at $\beta=0.71$, we have taken into account the ${ }_{35}$ Cryo-TEM results which show that at this stoichiometric ratio, the PE-S complex forms both wormlike and spherical (or short cylindrical) particles. In the proposed model, scattering from wormlike micelles, $P_{\text {worm }}\left(q, L, b, R_{\mathrm{c}}\right)$, is described by the form factor for the semiflexible chain with the contour length $L$ and the ${ }_{40}$ Kuhn length $b,^{32}$ assuming that the chain has a local cylindrical morphology with homogeneous scattering length density and the cross-section radius $R_{\mathrm{c}}$, while spherical particles are treated by the simple form factor $P_{\text {sphere }}\left(R_{\mathrm{s}}, q\right)$ for monodisperse spheres with the radius $R_{\mathrm{s}}$.

45 In addition to the scattering contributions from worms and spheres, it was necessary to treat correlations in the high $q$ region. The latter contribution was modelled by the structure factor for disordered cell-cell correlations, ${ }^{\mathbf{3 3}} S_{\text {cor }}(q, l, \xi)$, where $\xi$ is the correlation length. The overall scattering function, $I_{\mathrm{PE}-\mathrm{S}}(q)$, thus 50 has the form,

$$
\begin{aligned}
I_{\mathrm{PE}-\mathrm{S}}(q)= & I_{1} P_{\text {worm }}\left(q, L, b, R_{\mathrm{c}}\right)+I_{2} P_{\text {sphere }}\left(q, R_{\mathrm{s}}\right)+ \\
& +I_{3} S_{\text {cor }}(q, l, \xi)
\end{aligned}
$$

where $I_{1}$ and $I_{2}$, respectively, are the forward scattering intensities 55 for worms and spheres and $I_{3}$ is the amplitude of the correlation peak. (The full expression for $I_{\mathrm{PE}-\mathrm{S}}(q)$ is given as Supplementary Information.)

The resulting parameters of the fit of Eqn. (3) to the SAXS data for $\beta=0.71$ (shown in Fig. 3 as the solid line) with the fixed 60 contour length $L=600 \mathrm{~nm}$ and Kuhn length $b=200 \mathrm{~nm}$ are, $R_{\mathrm{c}}=$ $15.5 \pm 0.2 \mathrm{~nm}, R_{\mathrm{s}}=13.8 \pm 0.1 \mathrm{~nm}, l=3.67 \pm 0.02 \mathrm{~nm}$ and $\xi=$ $6.8 \pm 0.5 \mathrm{~nm}$, with $\chi^{2}=5.4$. The radii of the worms and spheres are consistent with the Cryo-TEM images, being only slightly larger than those obtained form the microscopic image. This 65 difference can be ascribed to the fact that hydrated aggregates or domains in the particles are not visible because of low electron contrast difference between these structures and amorphous ice of the solvent. The characteristic packing distance compares with the cross-section diameter of threadlike micelles. It should also be 70 pointed out that even though the scattering contribution from the spheres is relatively low $\left(I_{2} / I_{1}=1.7 \times 10^{-2}\right)$, it cannot be neglected as running the fit with the $I_{2}$ value fixed at $I_{2}=0$ (without the presence of spherical particles) results in a significantly higher $\chi^{2}$ value $\left(\chi^{2}=62.4\right)$, so that the SAXS data analysis corroborates the ${ }_{75}$ Cryo/TEM observation of spherical particles coexisting with the worms.

80

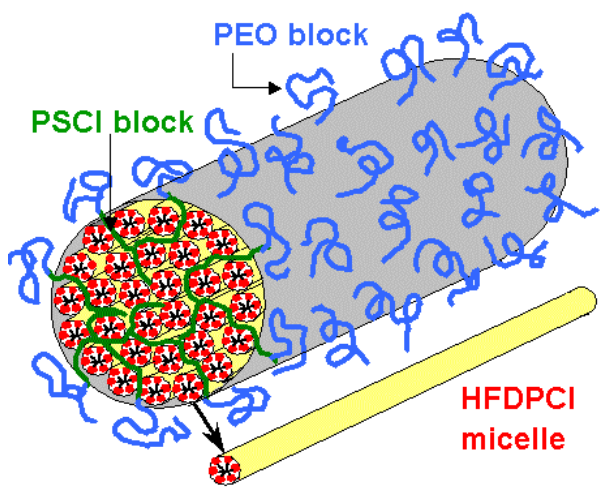

Fig. 5 Scheme of the PSCI-PEO/HFDPCl core-shell wormlike nanoparticle.

\section{Conclusion}

In this article, we have studied the interaction of a double hydrophilic anionic block polyelectrolyte, PSCI-PEO, with a cationic fluorosurfactant, $\mathrm{HFDPCl}$, in aqueous solutions, by a 95 combination of scattering (SLS, DLS, SAXS) and microscopic (Cryo-TEM, AFM) techniques. We have demonstrated that double hydrophilic block polyelectrolytes are able to form PE-S complexes with oppositely charged fluorosurfactants in a similar way as for PE-S complexes involving hydrocarbon ionic 100 surfactants, so that at the stoichiometric ratio between SCI units and $\mathrm{HFDPCl}$ corresponding to $\zeta$ potential values of the aggregates close to zero, the block polyelectrolyte co-assembles with the fluorosurfactant in compact core-shell nanoparticles with the core formed by the phase consisting of densely packed 
fluorosurfactant micelles and polyelectrolyte blocks and the shell of hydrophilic neutral blocks. In the case of PSCI-PEO/HFDPCl system, however, micellization of $\mathrm{HFDPCl}$ in threadlike, cylindrical micelles causes the formation of long wormlike core5 shell nanoparticles (coexisting with a small fraction of spherical PE-S nanoparticles), as was shown by cryogenic transmission electron microscopy and attested by SAXS measurements. The formed core-shell nanoparticles are apt to disruption if $\mathrm{HFDPCl}$ is added in excess with respect to the stoichiometry 10 corresponding to the zero effective charge.

SAXS data for the wormlike core-shell nanoparticles were treated by the model for semiflexible chains with the local cylindrical cross-section and homogeneous scattering length density, with an additional term taking into account the 15 correlations between densely packed surfactant micelles in the core of the nanoparticles. The structure of the wormlike nanoparticles proposed on the basis of SAXS and Cryo-TEM measurements is depicted in Fig. 5.

\section{${ }_{20}$ Acknowledgement}

The authors acknowledge the financial support from the Ministry of Education of the Czech Republic (long-term Research Project No. MSM0021620857), the Czech Science Foundation grants P208/10/0353 (M.Š.), P208/10/1600 (B.A.), P208/12/P236

25 (M.U.), P302/12/G157 (L.K.) and P205/11/J043 (K.P.) and from the Charles University grant UNCE204022 (L.K.). The authors thank Dr. M. Urbanová from the Institute of Macromolecular Chemistry of the Academy of Sciences of the Czech Republic for sample characterization by ${ }^{13} \mathrm{C}$ solid state NMR. B.A. 30 acknowledges a kind technical support from Manfred Rössle at the Petra III BioSAXS beamline.

\section{Notes and references}

${ }^{a}$ Department of Physical and Macromolecular Chemistry, Faculty of Science, Charles University in Prague, Hlavova 2030, 12840 Prague 2, 35 Czech Republic. Fax: +42022499752; Tel: +420221951292; E-mail: stepanek@natur.cuni.cz

${ }^{b}$ Institute of Macromolecular Chemistry, Academy of Sciences of the Czech Republic, Heyrovský Square 2, 16206 Prague 6, Czech Republic ${ }^{c}$ Institute of Cellular Biology, 1 st Faculty of Medicine, Charles

40 University in Prague, Albertov 4, 12801 Prague 2, Czech Republic

${ }^{d}$ Helmholtz-Zentrum Geesthacht, Centre for Materials and Coastal Research, D-21502 Geesthacht, Germany

${ }^{e}$ Theoretical \& Physical Chemistry Institute, National Hellenic Research Foundation, 48 Vassileos Constantinou Avenue, 11635 Athens, Greece.

45 Fax: +302107273824; Tel: +302107273794; E-mail: pispas@eie.gr

$\dagger$ Electronic Supplementary Information (ESI) available: (i) The CryoTEM micrograph of a HFDPCl micelle and (ii) the full expression for the scattering function $I_{\mathrm{PE}-\mathrm{S}}(q)$ (Eqn. (3)). See DOI: 10.1039/b000000x/

1 I. K. Voets, A. de Keizer and M. A. C Stuart, Adv. Colloids Interface $50 \quad$ Sci., 2009, 147-148, 300.

2 D. Langevin, Adv. Colloids Interface Sci,. 2009, 147-148, 170.

3 S. Zhou and B. Chu, Adv. Mater., 2000, 12, 545.

4 D. Chu and J.K. Thomas, J. Am. Chem. Soc., 1986, 108, 6270.

5 P. Hansson, Langmuir, 2001, 17, 4167.

556 K. Kogej, J. Phys. Chem. B, 2003, 107, 8003.

7 A.V., Kabanov, T.K. Bronich, V.A. Kabanov, K. Yu and A. Eisenberg, J. Am. Chem. Soc., 1998, 120, 9941.

8 T.K. Bronich, A.M. Popov, A. Eisenberg, V.A. Kabanov and A.V. Kabanov, Langmuir, 2000, 16, 481.
609 T.K. Bronich, A.V. Kabanov, V.A. Kabanov, K. Yu and A. Eisenberg, Macromolecules, 1997, 30, 3519.

10 J.-F. Berret, G. Cristobal, P. Hervé, J. Oberdisse and I. Grillo, Eur. Phys. J. E, 2002, 9, 301.

11 J.-F. Berret, B. Vigolo, R. Eng, P. Hervé, I. Grillo and L. Yang, 65 Macromolecules, 2004, 37, 4922.

12 J. Courtois and J.-F. Berret, Langmuir, 2010, 26, 11750.

13 K. Matsuoka and Y. Moroi, Current Opinion Colloid Interface Sci., 2003, 8, 227.

14 A. F. Thünemann, Langmuir, 1998, 14, 4898.

7015 C.K. Ober and G.Wegner, Adv. Mater., 1997, 9, 17.

16 A.F. Thünemann and K.H. Lochhaas, Langmuir, 1999, 15, 6724.

17 A.F. Thünemann, Prog. Polym. Sci., 2002, 27, 1473.

18 S.-S. Hou, J.-K. Tzeng and M.-H. Chuang, Soft Matter, 2010, 6, 409.

19 H. Peng, D. Chen and M. Jiang, J. Phys. Chem. B, 2003, 107, 12461.

7520 Z. Hu, W. Verheijen, J. Hofkens, A.M. Jonas and J.-F. Gohy, Langmuir, 2007, 23, 116.

21 A. Laschewsky, M. Mertoglu, S. Kubowicz and A.F. Thünemann, Macromolecules, 2006, 39, 9337.

22 S. Pispas, J. Polym. Sci. A Polym. Chem., 2006, 44, 606

8023 K. Wang, G. Karlsson, M. Almgren and T. Asakawa, J. Phys. Chem. B 1999, 103, 9237.

24 M. Uchman, M. Štěpánek, K. Procházka, G. Mountrichas, S. Pispas, M. Špírková and A. Walther, Langmuir 2008, 24, 12017.

25 M. Uchman, M. Štěpánek, K. Procházka, G. Mountrichas, S. Pispas,

85 I.K. Voets and A. Walther, Macromolecules, 2009, 42, 5605.

26 S. Pispas, J. Phys. Chem. B, 2007, 111, 8351.

27 S. Pispas, Soft Matter, 2011, 7, 474.

28 M. Karayianni, S. Pispas, G.D. Chryssikos, V. Gionis, S. Giatrellis and G. Nounesis, Biomacromolecules, 2011, 12, 1697.

9029 M. Uchman, K. Procházka, K. Gatsouli, S. Pispas and M. Špírková, Colloid Polym. Sci., 2011, 289, 1045.

30 T. Asakava, H. Hisamatsu and S. Miyagishi, Langmuir, 1995, 11, 478.

31 J. Dubochet, M. Adrian, J.J. Chang, J.C. Homo, J. Lepault, A.W. 95 McDowall and P.Q. Schultz, Rev. Biophys., 1988, 21,129.

32 J.S. Pedersen and P. Schurtenberger, Macromolecules, 1996, 29, 7602.

33 N. Lei, C.R. Safinya, D. Roux and K.S. Liang, Phys. Rev. E, 1997, 56, 608 .

100 